\title{
GCU
}

Glasgow Caledonian

University

University for the Common Good

\section{Active distribution power system with multi-terminal DC links}

Chen, Dong; Xu, Lie; Zhang, Wendian

Published in:

IET Renewable Power Generation

DOI:

10.1049/iet-rpg.2016.0154

Publication date:

2017

Document Version

Author accepted manuscript

Link to publication in ResearchOnline

Citation for published version (Harvard):

Chen, D, Xu, L \& Zhang, W 2017, 'Active distribution power system with multi-terminal DC links', IET Renewable Power Generation, vol. 11, no. 1, pp. 27-34. https://doi.org/10.1049/iet-rpg.2016.0154

\section{General rights}

Copyright and moral rights for the publications made accessible in the public portal are retained by the authors and/or other copyright owners and it is a condition of accessing publications that users recognise and abide by the legal requirements associated with these rights.

Take down policy

If you believe that this document breaches copyright please view our takedown policy at https://edshare.gcu.ac.uk/id/eprint/5179 for details of how to contact us. 


\title{
Active Distribution Power System with Multi-terminal DC links
}

\author{
Dong Chen* ${ }^{1}$, Lie $\mathrm{Xu}^{2}$, Wendian Zhang ${ }^{2}$ \\ ${ }^{1}$ Department of Engineering, Glasgow Caledonian University, Cowcaddens Road, Glasgow, \\ United Kingdom \\ ${ }^{2}$ Department of Electrical and Electronics Engineering, Strathclyde University, 204 George \\ Street, Glasgow, United Kingdom \\ *dong.chen@gcu.ac.uk; lie.xu@strath.ac.uk; wendian.zhang@strath.ac.uk
}

\begin{abstract}
A fast power restoration operational scheme and relevant stabilizing control is proposed for active distribution power systems with multi-terminal DC network in replacement of the conventional normal open switches. A 9-feeder benchmark distribution power system is established with a 4-terminal medium power DC system injected. The proposed power restoration scheme is based on the coordination among distributed control among relays, load switches, voltage source converters and autonomous operation of multi-terminal DC system. A DC stabilizer is proposed with virtual impedance method to damp out potential oscillation caused by constant power load terminals. The proposed system and controls are validated by frequency domain state space model and time domain case study with Matlab/Simulink.
\end{abstract}

\section{Nomenclature}

Fig. 1.

Fig. 2.

Fig. 3.

Fig. 4.

Fig. 5.

Fig. 6.

Fig. 7.

Fig. 8.

Fig. 9.

$C_{3} C_{6} C_{C} C_{G}$

$C_{v}$

$i_{\text {dref }}, i_{\text {qref }}$

$i_{d}{ }^{\prime}, i_{q}{ }^{\prime} i_{a b c}$ '

$i_{E S S}, i_{s t a}$

$i_{\text {Charg }}$

$i_{3}, i_{6}, i_{C}, i_{G}$

$I_{\text {fabc }}$

$i_{36}, i_{G 3}, i_{3 C}$

$L_{36}, L_{3 C}, L_{G 3}$

$P_{C}, P_{G}, P_{6}, P_{3}$

$R_{36}, R_{3 C}, R_{G 3}$

$R_{v}$

SCR

$T_{3}, T_{6}, T_{C}, T_{G}$

$U_{60}, U_{C 0}, U_{G 0}$

$V_{\text {dref, }}, V_{\text {qref }}$

$V_{d} V_{q}$

$V_{d c}$
Benchmark system of the distribution power system

Voltage control mode of voltage source converter

DC system modelling and control

Frequency response of charging station terminal

Eigenvalue distribution of the DC system

DC network control scheme

AC fault behaviour (Fault 1)

Stabilizer Performance (Charging Power; DC Voltage)

Fast power supply restoration after 3-phase fault

DC Terminal capacitance of VSC 3, VSC 6, charging station and generation

virtual DC capacitance

reference current of VSC in $d$, q axis

current detection of VSC in $d, q$ axis and abc reference frame

charging station current, stabilizer current

total current at charging station terminal

DC terminal currents of VSC 3, VSC 6, charging station and generation

AC fault current

DC line current of Line $36, G 3,3 C$

DC Line inductances of Line 36, 3C, G3

terminal powers of the charging station, generation, VSC 6, VSC 3

DC Line resistances of Line 36, 3C, G3

virtual resistance

short circuit ratio

current loop delay time constant of terminal VSC 3, VSC 6, charging station and generation terminal operational voltage of VSC 6, charging station, generation

$A C$ voltage reference values of $d$-axis and $q$-axis

$A C$ voltage in d-axis, $q$-axis

$D C$ voltage 


\begin{tabular}{ll}
\hline$V_{1}, V_{2}$ & AC voltage magnitudes across the load switch \\
$V_{N}$ & nominal AC voltage magnitude \\
$V_{3}, V_{6}, V_{C}, V_{G}$ & DC terminal voltages of VSC 3, VSC 6, charging station, generation \\
$V_{d c l} V_{d c 2}$ & DC threshold voltages for operational mode switching \\
$V_{f a b c}$ & AC voltage at fault point \\
$\omega_{r e f}, \omega_{N}$ & reference frequency, nominal frequency of a VSC \\
$\Delta \omega$ & frequency error of a VSC \\
$\boldsymbol{O}_{\mathbf{1}}, \boldsymbol{O}_{2}$ & AC voltage angles across the load switch \\
$\theta$ & VSC angle
\end{tabular}

\section{Introduction}

The development of distributed power generation [1] and advance of Electric Vehicle (EV) [2] are changing the configuration of the traditional power distribution system. Renewable power, such as wind [3] and solar [4], are naturally dispersed and compatible for distribution side. Comparing with large renewable generation, which are normally far away from load centre, power produced by distributed generation is more likely to be consumed locally and there is no need to build up extra high voltage corridor [5] to transmit bulk intermittent power to the load centre. However, mismatch between loads and generations is almost inevitable especially when a distribution substation is feeding both household and industrial load feeders at the same time as both load profiles fairly diverse [6]. Serious mismatch at "last mile" ends of a distribution power system can possibly give rise to severe voltage variations [7]. In some cases, possible over-voltage can reduce their nominal life spans or even damage power equipment, which can be caused by DG power that significantly overweighs local load at the far end from the distribution substation [7]. Such problem is difficult to solve with traditional passive distribution power system. Reactive power control based voltage regulation [8][9] might play a part to help but with limited performance in a more resistive distribution network. A pre-set renewable generation curtailment scheme may help, however, at a cost of certain amount of renewable energy loss.

The potential rising demand from the electric vehicle battery charging is also a serious challenge to the conventional distribution network as the charging load can potentially be much larger than traditional household and commercial appliances. With varying charging demand from EV, overloading and consequent contaminated voltage profiles could be the major problems for traditional distribution systems [10]. One possible solution to this problem is to build up centralised charging stations with a predefined energy management scheme and integrate them into the existing power system in a more grid-friendly way [11]. To charge an EV battery array with local renewable generation would be an ideal scenario [11].

In order to reduce the distribution loss and enhance power supply reliability, the concept of active distribution power system is proposed with load switch spread among certain feeders [12]. Such 
reconfigurable distribution power system is able to actively redistribute power flow by switching the ties between the feeders, hence the possibility of power flow optimization in terms of distribution losses and voltage profiles. A great variety of smart management methods have been proposed for optimized power flow management based on hard switches [13][14][15]. However, the employment of hard tie switches forces the voltages across different feeders to match each other, leading to inflexible power flow control since the real time power flow is passively determined by the impedance distribution of a certain system configuration pattern and the total number of patterns for a certain system is limted.

Though radial topology is most commonly used in distribution network for its simplicity feature [16], ring topology are also widely implemented, with Normal Open Switch (NOS) as loop breaking point [17][18], to ensure power supply restoration after a fault. However, the power restoration based on the switching involves NOS control can take minutes to complete [17][18]. Although the closing of NOS can easily increase system load availability with diverting power dispatching route, it would create a close-loop or even meshed distribution network with reduced fault impedances hence significantly increase fault currents. This could undermine the existing protection and relay system, which is unacceptable so far.

In order to cope with the challenges brought by higher DG and EV penetration to distribution power system, multi-terminal DC networks are proposed in distribution power system level, which is able to enhance voltage profile and load availability, and facilitate more flexible and faster power flow control [7][19] over emerging DGs and charging loads without significant contribution to fault current.

In this paper, a four-terminal DC network is proposed and inserted into a benchmark distribution power system to replace the conventional NOS. An AC-DC coordinating scheme is proposed for power supply restoration to minimize the outage time after a fault on AC feeder. To further take the advantage of the DC link, DG and charging station are also integrated within the DC network for cost and conversion efficiency concern.

One side effect brought about by the multiple-terminal DC network is the dynamics and stability issue. This is due to the fact that, in a multiple terminal DC system, the terminal impedances of slack terminals are normally well designed and damped [20] while the constant power terminals are not [21-24]. The terminal impedances of constant power terminal mainly depend on their own static operational (Power) point regardless of system stability, which can be undesirable to system dynamics and stability [21-24]. When all the DC terminals are closely located, which means the line impedances are limited, the undesirable impedances can be possibly accommodated by the slack terminals [20-24]. However, when 
there are considerable distribution distances, the slack terminals may not be able to accommodate them from the far ends. As a result, measures have to be taken at the power terminal side. In this paper a stabilizing method is also proposed to improve system dynamics from the power terminal side by modifying the large constant power terminal impedances. It can be directly implemented on accessible terminals by superimposition over its conventional control; for those inaccessible DC terminals, charging station for instance, a storage based DC physical stabilizer is proposed to implement the proposed control at the corresponding power terminal.

This paper is organized as follows. System modelling is introduced in Section 2 and the specific operational schemes and DC stabilization control are described in Section 3. Case study is then performed in Section 4 and finally the conclusion is drawn in Section 5.

\section{System Modelling}
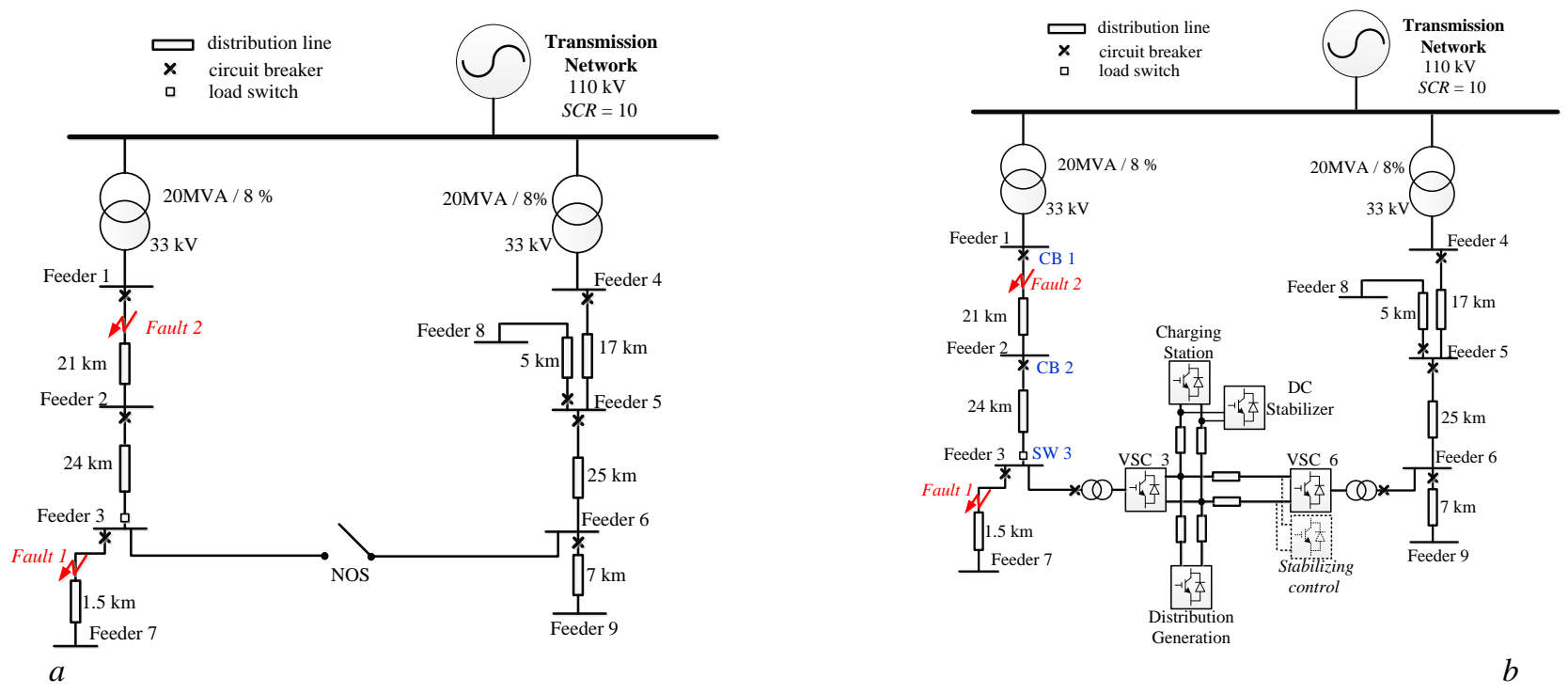

Fig. 1. Benchmark system of the distribution power system a without DC link

$b$ with DC link

In order to investigate the proposed active power distribution system, an original dynamic benchmark system is established, which is shown in Figure 1 a. As is shown, the distribution system in Figure 1 is based on a single ring topology with an NOS between Feeders 3 and 6 . Nine feeders are spreading all over the downstream side of the two distribution transformers rated at $20 \mathrm{MW}$ each at $35 \mathrm{kV}$ at the secondary side. The primary sides of the transformers are fed by the main power grid with rated voltage level at $110 \mathrm{kV}$ and the strength of the grid is defined with a Short Circuit Ration (SCR) of 10. Loads and DGs are connected to each feeder. Centralized passive load and DG are considered attached to 
each feeder. Closed-loop current controls are implemented within the DGs, which assumes that all the DGs are well capable to go through AC faults.

An example of the proposed active distribution power system is derived from the system demonstrated by Figure 1 a. By replacing the NOS with a multi-terminal DC link, as is shown in Figure 1b, an active distribution power system with flexible DC link is configured. Two VSCs, namely VSC 3 and VSC 6, are connecting to Feeder 3 and 6 respectively. A DC charging station and a centralized DC-side DG are connected to VSC 3 side via two DC lines. Both DC charging station and DG has a power rating of 2 MW. Both charging station and DG terminals are also with well-implemented closed-loop current control.

\section{Operational Scheme}

A distributed control and protection scheme is proposed for the active distribution power system with fast power supply restoration capability. The control units involve AC relays and its relevant circuit breakers (re-closers), load switches and all the converters connected to the DC network. The coordination is mainly based on local detections and reactions in the first place and, in addition, with a minimum level of communication.

The distribution power system operates with a centralised control based on low bandwidth communication, via which the amount of power passing through the DC network to is primarily determined by a distribution central controller during steady state.

As VSC control plays a key role in the distribution operation between AC and DC side, the possible operational modes of a VSC is introduced first. A virtual impedance based stabilizer is then proposed to stabilize the DC system. The stabilizer mainly concerns charging station, which can possibly spells instability as a variable constant-power type load. A DC voltage variation based autonomous power management scheme [20] is then introduced to cope with the fast dynamics of the DC system. Based on the above sub-sections, the procedure of post-fault power restoration is then proposed in this section.

\subsection{Voltage Source Converter modelling and control mode}

Predefined VSC voltage control strategies are considered in the proposed active distribution power system. Apart from the widely used synchronous current/power control mode [21], the VSCs are also able to operate in either DC voltage control or AC voltage control mode as is shown by Figures $2 \mathrm{a}$ and $2 \mathrm{~b}$ respectively. As is shown in Figure 2a, when a VSC is employed to control the DC voltage, a PI regulator is used to eliminate the difference between the reference DC voltage $V_{d c r e f}$ and $V_{d c}$. The output reference current in d-axis is fed to the current reference value responsible for the active power. The control scheme 
of AC voltage control is shown Figure $2 \mathrm{~b}$, where $V_{\text {dref }}$ and $V_{\text {qref }}$ are the reference values of the dq $\mathrm{AC}$ voltage components ( $V_{\text {qref }}$ is normally set at 0 for power decoupling concern) and $\omega_{\text {ref }}$ is the reference frequency. The currents of $i_{d}$ ' and $i_{q}$ ' are in-fed detections from the AC grid side.

And the VSC is also able to provide active resynchronization when reconnecting an AC island to an established utility side and the control scheme is depicted in Figure 2c, where the difference between utility side voltage angle $\theta_{1}$ and island side angle $\theta_{2}$ across the switch is passed to a PI regulator to produce the frequency correction to eliminate the angular difference; meanwhile a PI regulator is used to mitigate voltage differences between feeder side voltage $V_{1}$ and $V_{2}$ to avoid impact upon the switch close.
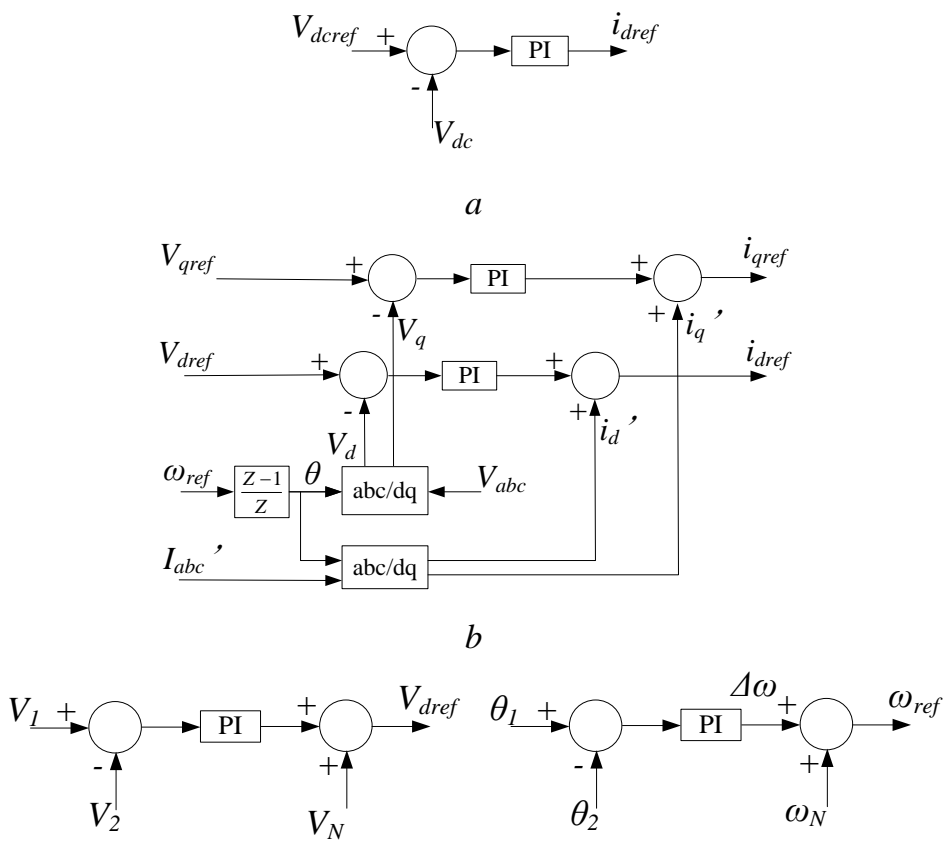

Fig. 2. Voltage control mode of voltage source converter

a DC voltage control

$b$ AC voltage control

c resynchronization control

\subsection{Charging station modelling and DC stabilizer control}

As a charging station is able to manage its own storage energy and provide temporary power support to the distribution power system, two modes are considered during normal operation, which is shown in Figure 3. In Figure 3a, the charging station operates as constant power mode with its current loop control modelled as a first order delay.

Large constant power terminal tends to introduce undesirable terminal impedance causing instability [22] especially from a distant position to the slack terminal. Reduced capacitance, which is favourable for cost, footprint and fault current limiting concern, would possibly further tense up this problem [21]. In 
other to deal with the possible instability caused by the undesirable impedance introduced by constant power, A DC stabilizer is proposed at the constant power terminal to neutralise the total equivalent terminal impedance at the constant power terminal side. An illustration is shown in Figure $3 \mathrm{c}$ with charging station terminal as an example. The principle of the proposed control is to add virtual parallel resistances at the power terminals with virtual impedance method, shown in Fig. 4, to cancel the undesirable terminal resistance (admittance). For instance, if the constant power load produce a negative admittance/resistance, a positive virtual resistance/admittance would be placed in parallel beside it. Meanwhile, virtual capacitance is also superimposed to the stabilizing control to further enhance the dynamic performance to add up to the reduced terminal capacitance at the constant power terminal side. As is shown in Fig. 3c, a physical DC stabilizer is installed beside the charging station for illustration. The proposed stabilizer can be a shunt converter with an energy storage, i.e. a battery or super capacitor, as its source. Cascaded closed-loop control is implemented with current loop as the inner loop. By mimicking the small signal behaviour of shunt capacitance and resistance with the outer loop shown in Figure $3 \mathrm{c}$, the stabilizer can effectively modify the terminal impedance in frequency domain, illustrate by Fig. 3c. The open-loop Bode plot corresponding to the charging station terminal in Fig. 3c is shown in Fig. 4..

The charging station terminal is used as a test example with a charging power at $2 \mathrm{MW}$ in Fig. 4 . The corresponding open-loop Bode plot frequency response is depicted in Figure 4. Before the proposed control is added, the negative admittance makes the phase behaviour starts from 90 degrees lead, which is undesirable. By using the proposed control, the phase is modified with virtual resistance to start from 90 degrees lag as shown and the gain is also lowered partially owing to virtual capacitance with larger gain margin. With this case shown in Fig. 4, it demonstrates that the proposed control with virtual impedance can effectively provide satisfactory terminal impedance with decent margins when a large power constant power load is in place with relatively small terminal capacitance. A high-pass filter with large time constant, i.e. $5 \mathrm{~s}$, is added before the $i_{\text {sta }}$ is sent to the stabilizer to ensure the average current/power consumption of the stabilizer is zero. As shown in Figure 3c, as VSC control is assumed to be accessible by system operator, similar control can also be incorporated as a super-imposed component for the VSC in constant power load mode, VSC 6 for instance, if necessary.

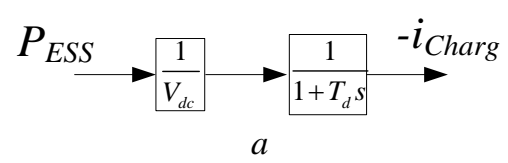




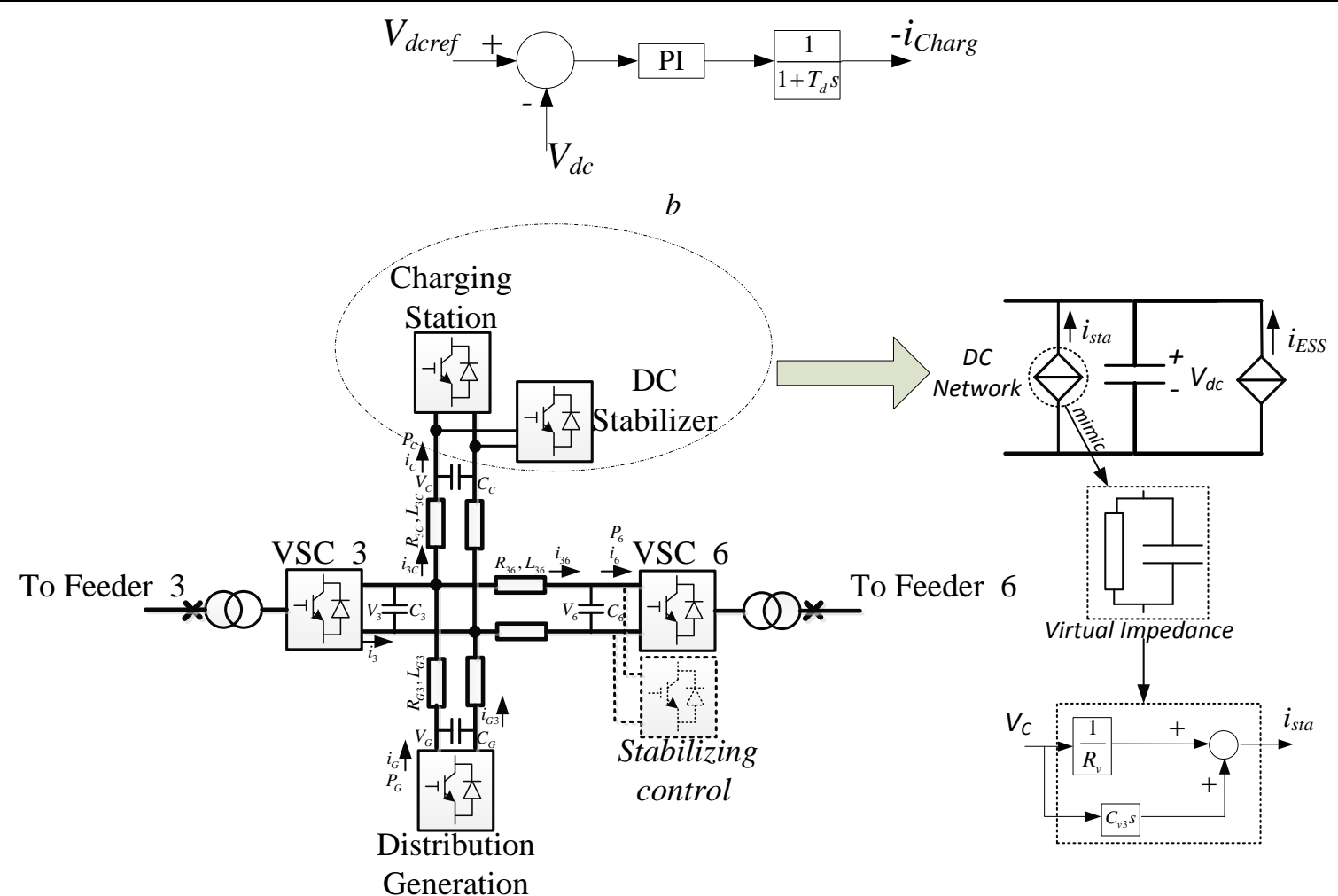

Fig 3. DC system modelling and control

a power control

b DC voltage control

c DC stabilizer

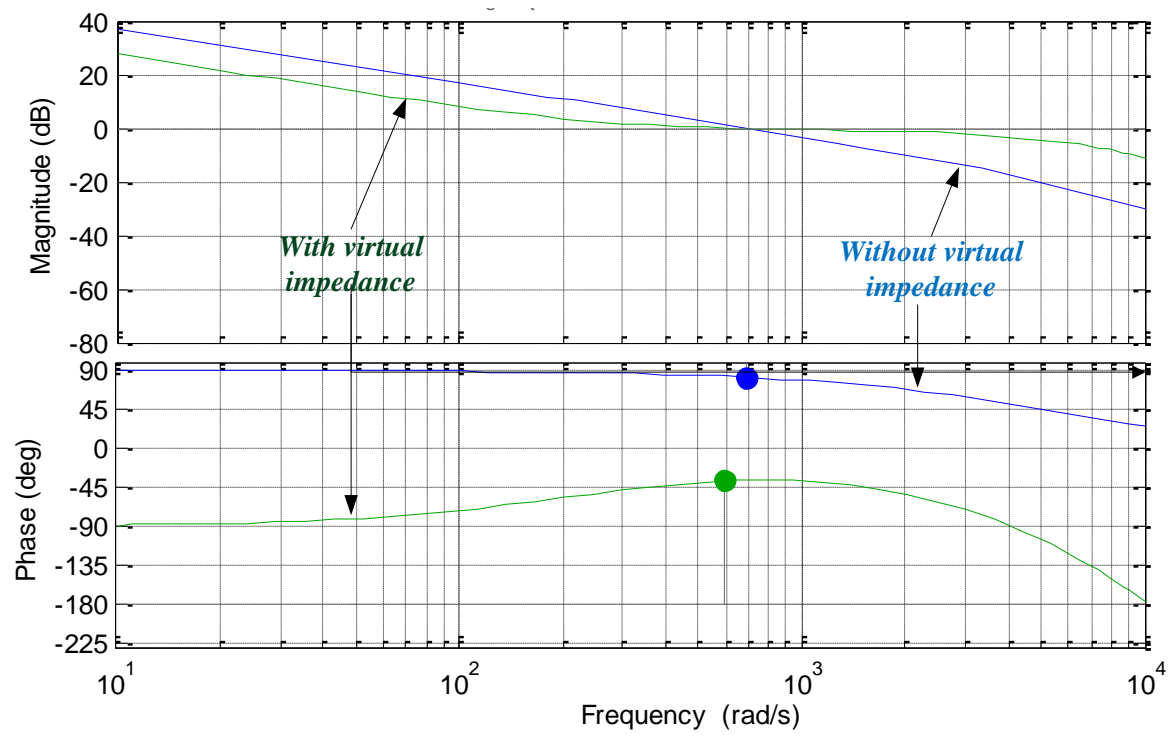

Fig. 4. Open-loop frequency response of charging station terminal

A small-signal state-space model is also established based on Figure $3 \mathrm{c}$ as follows to further analyse the stability of this system with constant power terminal stabilizing controls. Since the focus of this paper 
is placed on the interaction between constant power and DC voltage controls, the current loops are modelled as first order delays [25] for simplicity. As is shown in (1), a state space model is established with current loop delay time constants of VSC $3, \operatorname{VSC} 6$, generation and charging station defined as $T_{3}, T_{6}$, $T_{G}$ and $T_{C}$ respectively.

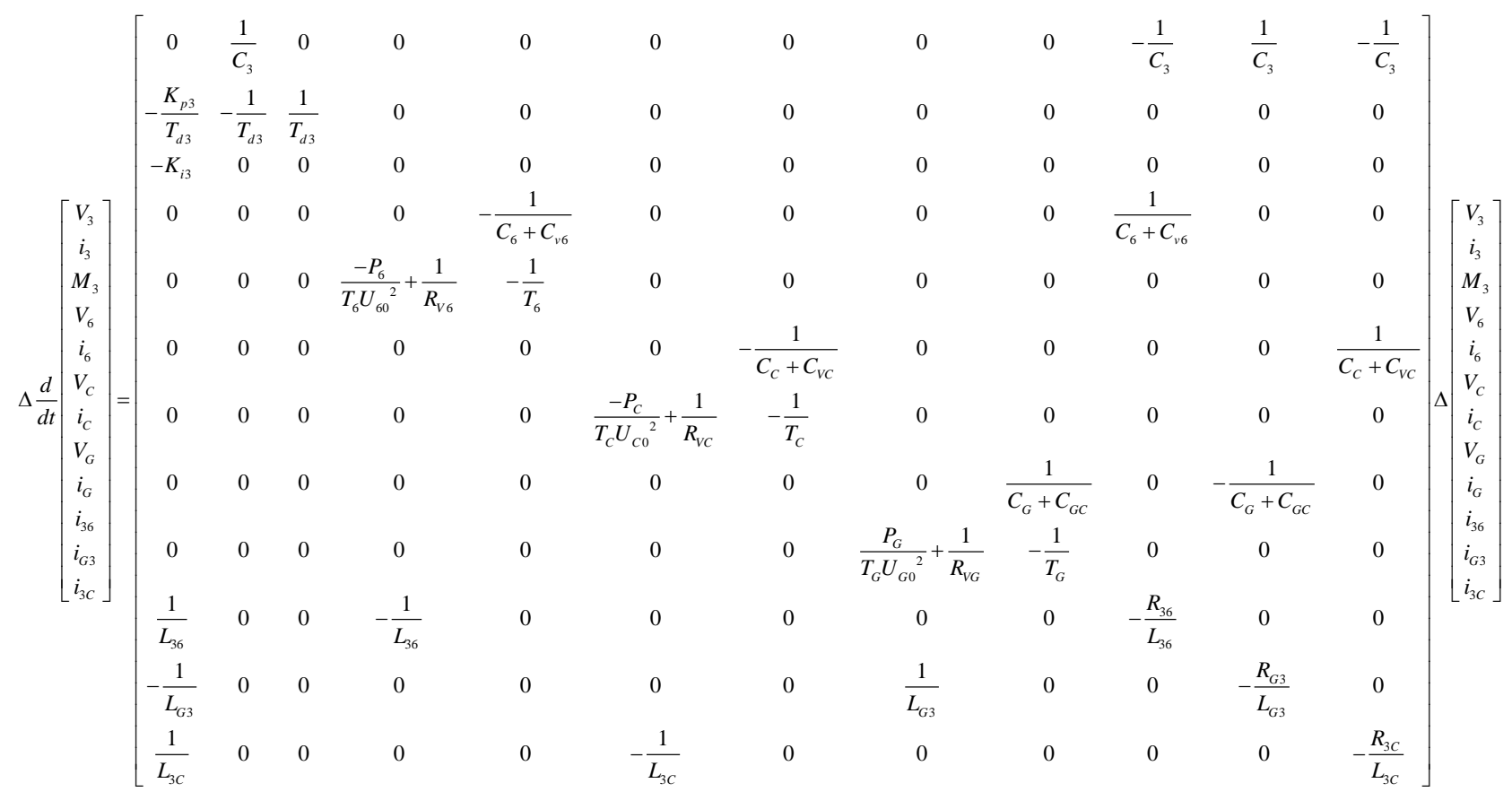

Based on the case shown in Fig. 4, the virtual impedance method control is added to the charging station at full power charging state while VSC 3 and generation terminals are with zero power for simplicity. The eigenvalues of the DC system is shown in Figure 5.

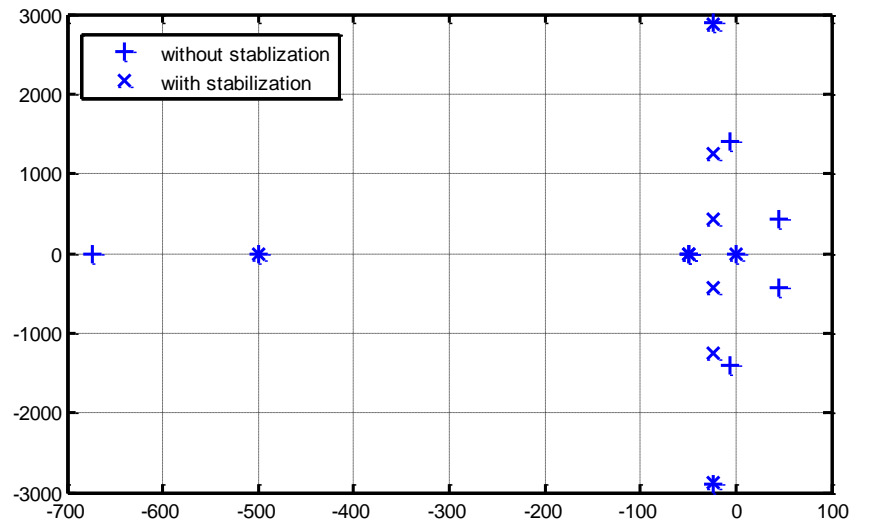

$a$

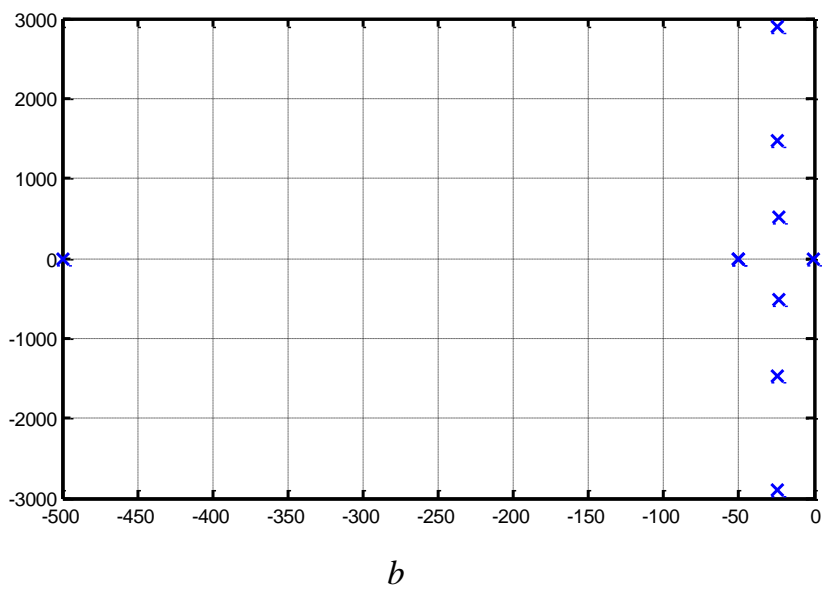

Fig. 5. Eigenvalue distribution of the DC system

$a P_{3}=0, P_{C}=2 M W, P_{G}=0$

$b P_{3}=-2 M W, P_{C}=2 M W, P_{G}=0$ 
The eigenvalues distributions are shown in Fig. 5 with the initial parameters setting listed in the appendix. In Fig. 5a, it shows that the added virtual impedance can well push the eigenvalues on the right panel into the left side and stabilize a DC system with full rating constant power load. Fig. 5b illustrate the situation when a large rectifying power is imported from VSC 3 based on the settings on Fig. 5 a, which demonstrate that the system can also well maintain stability when there is a large rectifying power. The above analysis validates the principles of the proposed virtual impedance control.

\subsection{DC network operational scheme}

A voltage variation based autonomous control scheme is proposed for the DC subsystem [20], which is shown in Figure 6. The DC power system has to have at least one live slack terminal to ensure stable operation. In this paper, VSC 6 is responsible to respond to the power distribution order given by the distribution system central controller and it operates at power control mode throughout steady state. When there is a fault at the AC side, VSC 6 may need to operate at current limit mode to ride through the fault. VSC 3 acts as the slack terminal during steady state operation, accommodating the power mismatch among VSC 6, charging station and DG with DC voltage control mode demonstrated in Figure 3b.

During an AC fault, VSC 3 might not be able to maintain DC voltage and will operate either at current limiting or AC voltage control mode hence effectively becomes a power terminal for DC network. The charging station terminal acts as a power terminal during normal steady state, managing its battery energy in its due course. When there is an AC fault, VSC 3 would lose its slack terminal capability and the DC voltage will deviate to level 2+/2-. The charging station terminal will then take over the role of slack terminal (assuming there is sufficient power rating and energy stored) once it detects the voltage has reached the threshold value, either $V_{1}$ or $-V_{2}$ and maintain the voltage at this point. The DG would take over the role of slack terminal if the charging station is fully charged as if the voltage would goes up into Level $3+$ zone. It is worth noting that the PI regulator for charging station voltage control is only valid when the voltage deviation is out of level 1. This design enables the VSC 3 autonomously re-taking over the DC voltage control back to level 1 without fighting with the charging station. Hysteresis loops may be employed for level switching to avoid undesirable oscillations. 


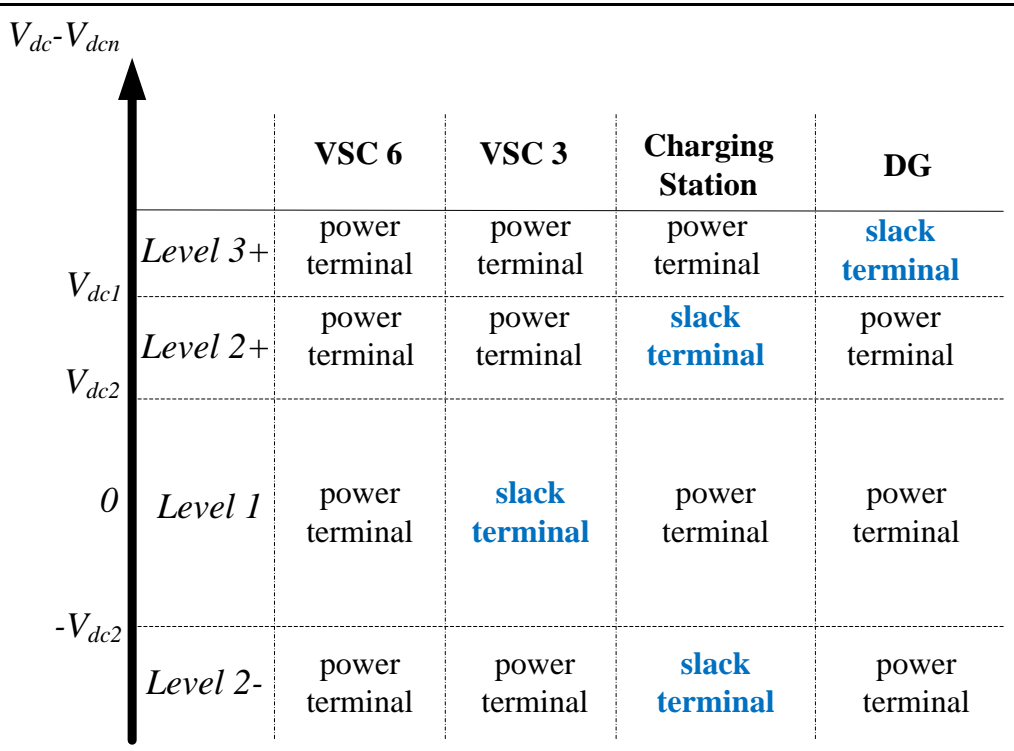

Fig. 6. DC network control scheme

\subsection{Post fault power restoration procedure}

The principle of the restoration is based on the coordination between DC operation and AC relays. When an AC fault occurs within the distribution power system, the VSCs will automatically operate in Fault Ride Through (FRT) mode and the relays shall isolate the fault. Immediately the fault is isolated, the downstream island power supply shall be restored by the VSC within. The CB shall reclose to check if the fault is cleared. Once the fault is cleared, the fault side VSC shall re-synchronize the island with its upstream and then re-connect the island back to the main grid. The DC autonomous control scheme shall apply throughout the process.

Based on the proposed restoration principles, a detailed operational procedure is designed for Fault 2 in Figure $1 \mathrm{~b}$ for illustration.

When an 3-phase short fault occurs at Fault 2 in Figure 1b, voltage dips and fault current will be immediately detected by both VSCs 3 and 6, and corresponding upstream relays respectively. The VSCs may do their best to keep their previous operational condition, unless the current limits have been reached, to ride through the fault. If the current limit has been reached, VSC 3, which is previously responsible for DC voltage control, will become a power terminal. This would give rise to DC voltage deviation and the relevant DC system mode switching scheme will apply as is cited in the previous sub-section and illustrated by Figure 5. The upstream relay shall also respond to the fault by open the upstream CB 1 .

Once the fault is cut off from the main distribution network, VSC 6 which is not cut off shall detect the local AC voltage is recovered and its capped power may be restored to what it was before the fault if 
applicable. This may give rise to another deviation of DC voltage and relevant DC mode switching scheme would again apply.

Once CB 2 is open, signals are passed to open CB 3 and SW 3 to isolate the fault. Upon receiving the signal, SW 3 passes the isolation signal to VSC 3 for islanding operation preparation. VSC 3 switches to AC voltage control mode shown in Figure $2 \mathrm{~b}$, hence the islanded Feeder 3 power supply is restored. This mode switch of the VSC may give rise to one more time of DC voltage deviation as the VSC power exchange with the DC side may change. Again, the DC network control scheme applies.

After a pre-set amount of time, CB 2 is reclosed. If the fault is not cleared, the CB is opened again. The procedure ends here till manual clearance of the fault. If the fault is not detected, the procedure proceeds.

Once CB 2 is successfully reclosed, the message is passed to its downstream load switch and VSC 3 within the island. The voltages across SW 3 are prepared to be resynchronized. The angular difference and voltage difference are detected across the switch and passed to VSC 3. Resynchronization operation is carried out with the scheme shown in Figure 2 c. Once both sides are confirmed synchronized, SW 3 is closed. Detecting the closing signal, VSC 3 is switched back to its pre-fault operation mode. Again, possible DC voltage deviation may be triggered hence relevant DC control operation performed.

The power restoration after an AC distribution fault can be summarized as follows:

1) Detecting AC fault

2) VSC 3 and 6 FRT

3) Distribution current relay responds to the fault

4) Upstream CB 1 open

5) CB 2 open

6) SW 3 open

7) VSC at islanding side switched to AC voltage mode

8) CB 1 reclose

9) If the fault is detected again, then CB 1 open; the procedure is ended waiting for manual clearance.

If the fault is not detected, the procedure proceeds.

10) Synchronization across SW 3.

11) SW 3 close

12) VSC 3 back to DC voltage control mode 


\section{Case Study}

\subsection{Feeder fault behaviour}

Based on the benchmark system demonstrated in Figure 1, a comparison case study is carried out to demonstrate the transient behaviour of the distribution power system after a 3-phase short between Feeders 6 and 7 shown in Figure 1a. It can be seen from Figure 6a that when there is a 3-phase fault, the fault current would go to approximately 3 p.u. at its peak value when there is a proposed DC link in the benchmark system; whereas in Fig. 7b, the Feeder 3 and 6 are connected by closed AC link, the corresponding fault current would rise up to about 5 p.u. which is $66 \%$ larger than the proposed system.
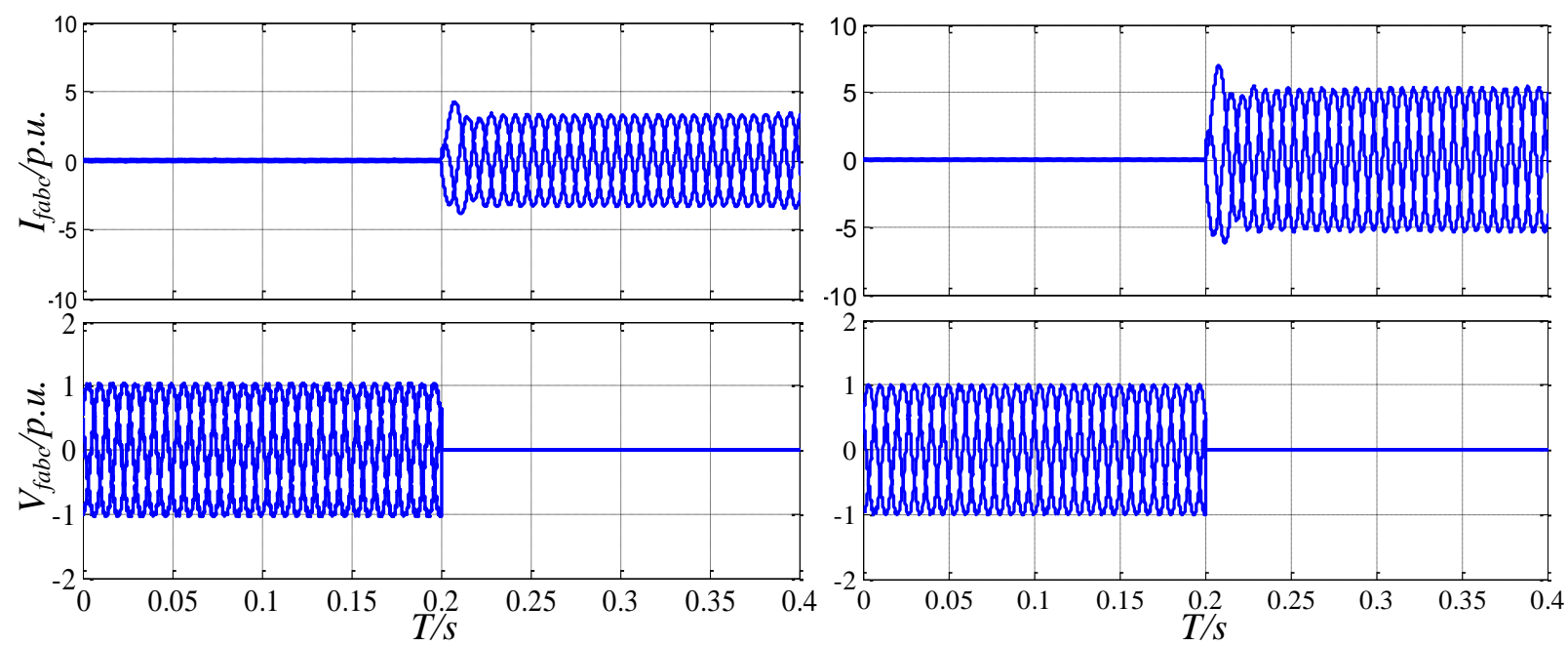

$a$

Fig. 7. AC fault behaviour (Fault 1)

a with DC link

$b$ with AC link

\section{2. $D C$ power stabilizer performance}

In order to validate the effectiveness of the proposed DC stabilizer, an operation test is carried out with focus on the stability of the multi-terminal DC system in Figure 8. During the test, a ramp power demand is given for the charging station from the time of $T=0.5 \mathrm{~s}$ at a ramp rate of $0.25 \mathrm{MW} / \mathrm{s}$ and the powers of VSC 6 and DG are kept at 0 . The DC stabilizer is activated from the beginning of the test till $\mathrm{T}$ $=5.5 \mathrm{~s}$ when it is deactivated. It can be seen that as soon as the proposed DC stabilizer is deactivated, the DC voltage starts to oscillate immediately hence the effectiveness of the proposed stabilizing control. 


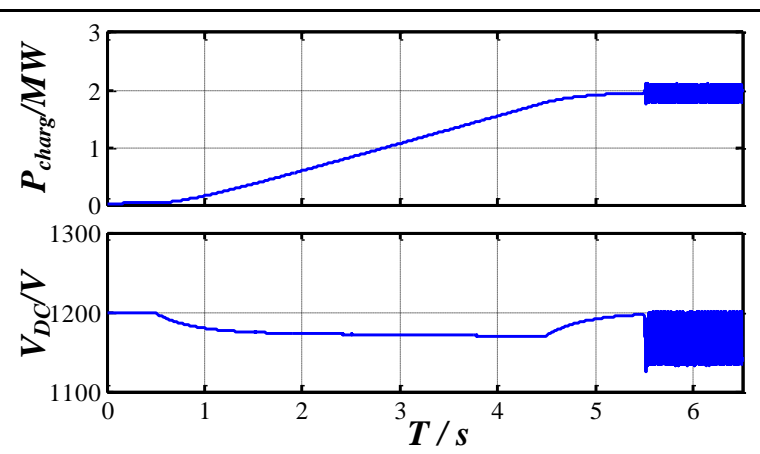

Fig. 8. Stabilizer Performance (Charging Power; DC Voltage)

\subsection{Fast power supply restoration}

An AC fault isolation and regional power supply fast restoration test is performed as the process shown in Table I and the corresponding simulation result is shown in Figure 9. In this test, the active distribution power system depicted in Fig. 1b operated at steady state at $T=0 \mathrm{~s}$. The VSC 6 operates as a constant power type terminal and VSC 3 the slack terminal at DC side. The charging station power is at 0. At (1) $T=0 \mathrm{~s}$, VSC 6 starts to import power from Feeder 6 at a ramp of $0.5 \mathrm{MW} / \mathrm{s}$ and to balance the DC system power, VSC 3 starts to export power $\left(P_{D C 3}\right)$ to Feeder 3 accordingly under DC voltage control mode.

At (2) $\mathrm{T}=2 \mathrm{~s}$, a three-phase short occurs at lower stream side of Feeder 1 (Fault 3) and the voltage at Feeder $3\left(V_{3}\right)$ drops to almost 0 immediately, the voltage of Feeder $6 V_{6}$ to 0.83 p.u.. With transient power fluctuation, the VSC 3 power $P_{\mathrm{DC} 3}$ becomes almost 0 as the $\mathrm{AC}$ voltage is almost 0 and with current capped at its rating; meanwhile, VSC 6 is still able to import power with a degraded rating. As the DC side lost the slack terminal VSC 3, the DC voltage at VSC 3 terminal $\mathrm{V}_{\mathrm{dc} 3}$ shoot up. As a result, the charging station starts to take over the DC voltage control as predefined when it detects the abnormal voltage transient of DC voltage and accommodate the imported power from VSC 6, shown by $P_{\text {charg }}$ and the DC voltage is maintained at $110 \%$ of nominal DC voltage as $1320 \mathrm{~V}$. As the distribution lost a part of its main load, the voltage of $V_{6}$ rises up slightly comparing with the stage before the fault. At (3) $\mathrm{T}=2.2 \mathrm{~s}, \mathrm{CB} 1$ is open and the voltage of Feeder $6 V_{6}$ is restored. At (4) $T=2.3 \mathrm{~s}, \mathrm{CB} 2$ is open, the fault is isolated from the downstream side as well. VSC 3 starts to restore the Feeders 3 voltage $V_{3}$ by operating as an AC voltage regulator at (5) $T=2.35 \mathrm{~s}$. At $T=2.4 \mathrm{~s}$, the load switch of Feeder 3 is open after the fault is cleared. A synchronization check is performed thereafter to tell if the upper stream voltage is restored and if yes then VSC 3 is informed the voltage and angular difference between both sides of the switch. At (7) $T=4 \mathrm{~s}$, this temporary fault is cleared itself. At (8) $T=4.2 \mathrm{~s}$, the circuit breaker at Feeder 1 is reclosed, which cause a 
slight voltage variation at Feeder 8 . At $9 T=4.3 \mathrm{~s}$, the circuit breaker at Feeder 2 is reclosed so voltage on Feeder 2 is restored. Upon being informed the voltage restoration of the upstream side, VSC 3 starts to synchronize both sides of load switch of Feeder 3, which causes minor voltage variation on Feeder 3. At (10 $T=5 \mathrm{~s}$, the load switch of Feeder 3 is closed and VSC returns to its slack terminal mode and re-take over DC voltage regulation. This transition causes a DC voltage shoot-up and both the stabilizer contributed to suppress it. At (11) $T=5.5 \mathrm{~s}$, VSC 6 decreases the import power from Feeder 6 at a ramp of $-0.5 \mathrm{MW} / \mathrm{s}$ until it reaches 0 . And at $T=10 \mathrm{~s}$, the charging station starts to consume power at a ramp of 0.5 $\mathrm{MW} / \mathrm{s}$ until it reaches $2 \mathrm{MW}$.

A slight power overshoot can be seen at (3) when the AC voltage is recovered back to normal from zero. This is due to a transient slight voltage overshoot of around $10 \%$. It causes a mismatch between the voltage and current when current is capped at its rated value. As the current can always be well controlled within a predefined limit, the overvoltage will not damage the switching device as long as it is below a certain voltage level (normally at a maximum of $140 \%$ for $1.2 \mathrm{kV}$ systems [26]). Practically, the transient overvoltage can be further well capped by dumping resistances if it is above a predefined voltage level [27]; the terminal transient power can therefore be capped regardless of the initial power input. Since the transient is very short, the total energy is limited. Such amount of energy can be well absorbed by the line impedances and terminal capacitances with limited voltage variation on other terminals; hence the impact to other converter control is trivial.

From the test shown in Table 1 and Figure 9, a fast power supply restoration scheme for Feeder 3 is performed. Similar scheme can also be carried out for other possible faults. The test shown in Figure 8 demonstrates that a multi-terminal DC system, with the proposed virtual impedance based stabilizer, connecting AC feeders and charging station can stably provide flexible power dispatch and fast power restoration after AC fault with large power redistribution over an expanded distribution area.

Similar power supply restoration scheme generally applies to the fault locations between a VSC feeder and an upstream feeder, which include Line 12, Line 23, Line 45 and Line 56 in Fig. 1b in this benchmark system. When the upstream connection is open, the feeder supply can be restored by a VSC as long as there is a distribution path between the feeder and the VSC. The faults at Line 37 and Line 58 cannot be applied since the protections will isolate Feeders 7 and 8 along with the fault apart from the VSCs.

Table 1 Fault isolation and fast power supply restoration operation (Fault 2) 

(1) $T=0 \mathrm{~s}$
VSC 6 starts to import power from Feeder 6 at a ramp of $0.5 \mathrm{MW} / \mathrm{s}$ until it reaches $1 \mathrm{MW}$
(2) $T=2 \mathrm{~s}$
3-phase short fault happens at upstream side of Feeder 2; PV start to generate power at a ramp rate of $0.5 \mathrm{MW} / \mathrm{s}$ till $0.5 \mathrm{MW}$

(3) $T=2.2 \mathrm{~s}$

CB 1 is opened

(4) $T=2.3 \mathrm{~s}$

CB 2 is opened

(5) $T=2.35 \mathrm{~s}$

VSC 3 starts to restore AC power supply at Feeder 3

(6) $T=2.4 \mathrm{~s}$

SW 3 at upstream of Feeder 3 is opened for resynchronization preparation

(7) $T=4 \mathrm{~s}$

Fault Clear

(8) $T=4.2 \mathrm{~s}$

Circuit breaker of Feeder 1 is reclosed

(9) $T=4.3 \mathrm{~s}$

Circuit breaker of feeder 2 is reclosed

(10) $T=5 \mathrm{~s}$

Load switch of Feeder 3 is closed; PV start to decrease power generation at a ramp rate of $-0.5 \mathrm{MW} / \mathrm{s}$ till 0

(11) $T=5.5 \mathrm{~s}$

VSC 6 decrease the import power from Feeder 6 at a ramp of $-0.5 \mathrm{MW} / \mathrm{s}$ until it reaches 0

(12) $T=10 \mathrm{~s}$

The charging station starts to consume power at a ramp of $0.5 \mathrm{MW} / \mathrm{s}$ until it reaches $2 \mathrm{MW}$

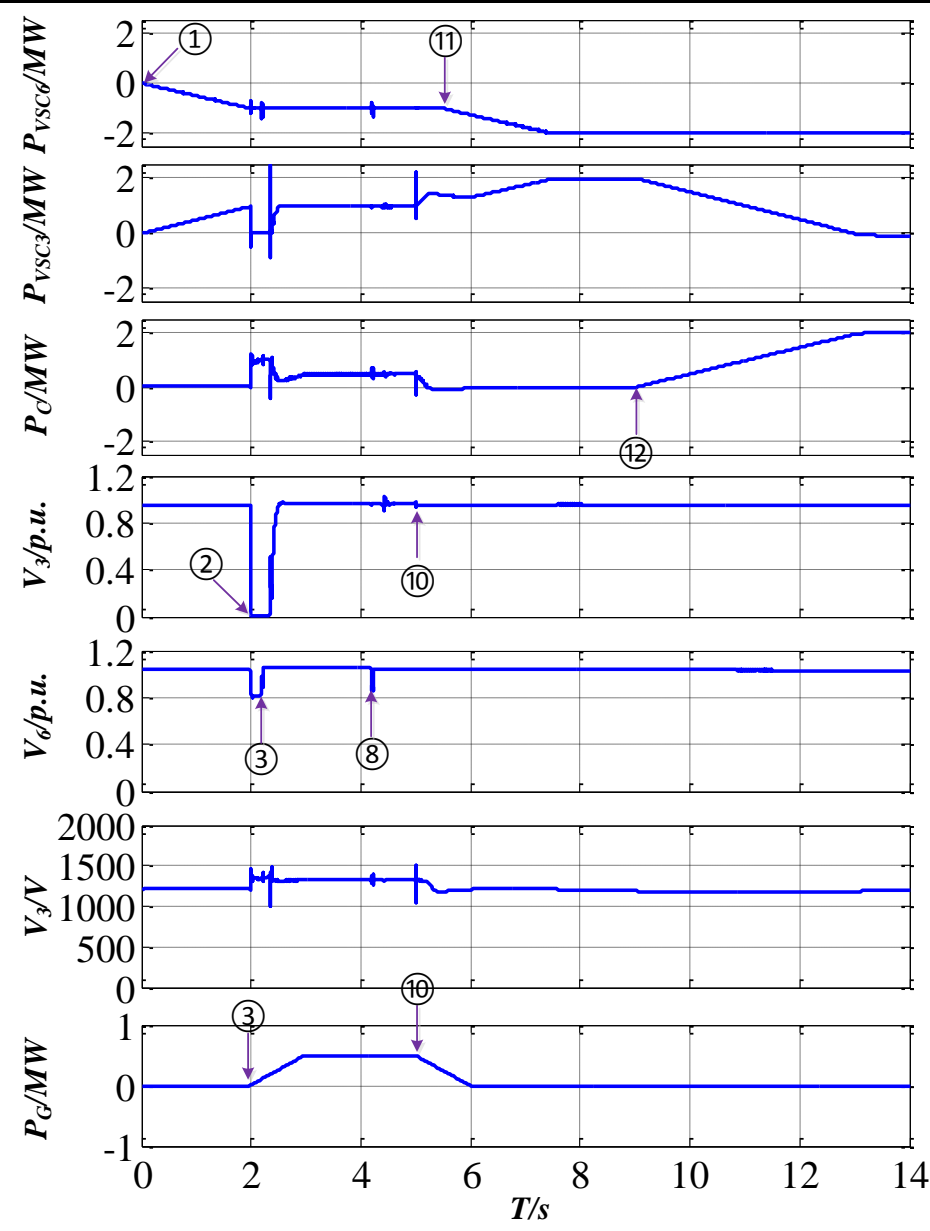

Fig. 9. Fast power supply restoration after 3-phase fault ((Power flowing out of DC system via VSC 6; Power flowing out of DC system via VSC 3; Charging power to charging station; Voltage of Feeder 6; Voltage of Feeder 3; DC voltage of VSC 3; $P V$ power generation)) 


\section{Conclusion}

In this paper a fast power supply restoration control scheme and corresponding DC stabilization control is proposed for an active distribution system with multi-terminal DC network replacing normal open switch. With proposed network, the flexible power distribution can be achieved without significant fault current contribution as a direct AC link does. The proposed stabilization control is able to stabilize the DC system with large constant power load type terminal and ensure a stable operation when providing power support to large AC transients. The proposed control scheme can effectively provide immediate power supply restoration after an $\mathrm{AC}$ fault and reconnect the islanding part back to the main grid if the fault is temporary and cleared within reclosing time set.

\section{Acknowledgments}

This work was supported in part by the UK Engineering and Physical Sciences Research Council: Grant EP/K03619X/1.

\section{References}

[1] Lasseter, R.: 'Microgrid'. IEEE PES Winter Meeting, Jan 2002, pp. 305-308

[2] Chan, C.C.: ' An overview of electric vehicle technology ', Proc. IEEE, 1993, 81, (9), pp. 1202-1213

[3] Atwa, Y.M., El-Saadany, E.F., Guise, A.-C.: ' Supply Adequacy Assessment of Distribution System Including Wind-Based DG During Different Modes of Operation ', IEEE Trans. Power Syst., 2010, 25, (1), pp. 78-86

[4] Karki, R., Billinton, R.: ' Reliability/cost implications of PV and wind energy utilization in small isolated power systems ', IEEE Trans. Energy Conv., 2001, 16, (4), pp. 368-373

[5] Bresesti, P., Kling, W.L., Hendriks, R.L., et al.: ' HVDC Connection of Offshore Wind Farms to the Transmission System ', IEEE Trans. Energy Conv., 2007, 22, (1), pp. 37-43

[6] Alcala, J., Cardenas, V., Perez-Ramirez, et al.: ' Improving power flow in transformers using a BTB converter to balance low voltage feeders ', Proc. Energy Conversion Congress and Exposition (ECCE), 2012, pp. 2038-2044

[7] Barragan, M., Mauricio, J.M., Marano, A., et al.: ' Operational benefits of multiterminal DC-links in active distribution networks ', Proc. IEEE Power and Energy Society General Meeting, July 2012, pp. 1-6

[8] Mithulananthan, N., Canizares, C.A., Reeve, J., et al.: ' Comparison of PSS, SVC, and STATCOM controllers for damping power system oscillations ', IEEE Trans. Power Syst., 2003, 18, (2), pp. 786-792

[9] Dahal, S., Mithulananthan, N., Saha, T.K.: ' Assessment and Enhancement of Small Signal Stability of a Renewable-Energy-Based Electricity Distribution System ', IEEE Trans. Sustain. Energy, 2012, 3, (3), pp. 407-415 
[10] Hajimiragha, A., Canizares, C.A., Fowler, M.W., et al.: ' Optimal Transition to Plug-In Hybrid Electric Vehicles in Ontario, Canada, Considering the Electricity-Grid Limitations ', IEEE Trans. Ind. Electron., 2012, 3, (3), pp. 407-415

[11] Liu, N., Chen, Q., Lu, Xinyi, et al.: ' A Charging Strategy for PV-Based Battery Switch Stations Considering Service Availability and Self-Consumption of PV Energy ', IEEE Trans. Ind. Electron., 2015, 62, (8), pp. 48784889

[12] Wu, J. S., K. Tomsovic, L., Chen, C. S.: ' A heuristic search approach to feeder switching operations for overload, faults, unbalanced flow and maintenance ', IEEE Trans. Power Del., 1991, 6, (4), pp. 1579-1585

[13] Qin, Z., Shirmohammadi, D., Liu, W.-H.E.: ' Distribution feeder reconfiguration for service restoration and load balancing ', IEEE Trans. Power Syst., 1997, 12, (2), pp. 724-729

[14] Ahmadi, H., Marti, J.R.: ' Distribution System Optimization Based on a Linear Power-Flow Formulation ', IEEE Trans. Power Del., 2015, 30, (1), pp. 25-33

[15] Khushalani, S., Solanki, J.M., Schulz, N.N.: ' Optimized Restoration of Unbalanced Distribution Systems ', IEEE Trans. Power Del., 2007, 22, (2), pp. 624-630

[16] Baran, M.E., Wu, F.F.: ' Network reconfiguration in distribution systems for loss reduction and load balancing ', IEEE Trans. Power Del., 1989, 4, (2), pp. 1401-1407

[17] UK Power Network -, 'Long Term Development Statement Network Summary 2012' (London Power Networks plc, 2012), pp. 8-14

[18] EDS 08-0150: 'London 33kV Design and Customer Supplies', 2014

[19] Rudion, K., Orths, A., Styczynski, Z.A, et al.: ' Design of benchmark of medium voltage distribution network for investigation of DG integration ', Proc. IEEE Power Engineering Society General Meeting, 2006

[20] Chen, D., Xu, L.: ' Autonomous DC Voltage Control of a DC Microgrid With Multiple Slack Terminals ', IEEE Trans. Power Syst., 2012, 27, (4), pp. 1897-1905

[21] Kazmierkowski, M. P. and Malesani, L.: ' Current control techniques for three-phase voltage-source PWM converters: a survey ', IEEE Trans. Ind. Electron., 45, no. 5, pp. 691-703, 1998.

[22] Marx, D., Magne, P., Nahid-Mobarakeh, B., et al.: ' Large Signal Stability Analysis Tools in DC Power Systems With Constant Power Loads and Variable Power Loads-A Review ', IEEE Trans. Power Electron., 2012, 27, (4), pp. 1773-1787

[23] Chen, D. and Xu, L., ' DC microgrid dynamic performance assessment and enhancement based on virtual impedance method ', Proc. IECON 2014 - 40th Annual Conference of the IEEE Industrial Electronics Society, 2014, pp. 1363-1369.

[24] Gu, Y., Li, W. and He, X.: ' Passivity-Based Control of DC Microgrid for Self-Disciplined Stabilization ', IEEE Trans. Power Syst., 30, no. 5, pp. 2623-2632, Sept. 2015.

[25] Prieto-Araujo E., Egea-Alvarez A., Fekriasl S. and Gomis-Bellmunt O., "DC Voltage Droop Control Design for Multiterminal HVDC Systems Considering AC and DC Grid Dynamics," IEEE Trans. Power del., vol. 31, no. 2, pp. 575-585, Jan. 2016. 
[26] 'Technical Information of IGBT-Module FZ3600R17HP4',

http://www.infineon.com/dgdl/Infineon-FZ3600R17HP4-DS-v02_02-en_de.pdf?fileId=db3a30432313ff5e0123560

$1 \mathrm{c} 5 \mathrm{db} 1610$, accessed 10 July 2016

[27] Olowookere, O., Skarvelis-Kazakos, S., Habtay, Y. and Woodhead, S.: ' Fault ride through during loss of converter in a 4-VSC based HVDC transmission ', Proc. IEEE PES Innovative Smart Grid Technologies, Istanbul, Turkey, 2014, pp. 1-6.

Appendix - The distribution power system parameters:

$U_{n}: 33 \mathrm{kV}$

$S_{b}: 20 \mathrm{MW}$

VSC 3: $2 \mathrm{MW}$

VSC 6: 2MW

Charging Station: $2 \mathrm{MW}$

DC side DG: $2 \mathrm{MW}$

DC line: $7.2 \mathrm{~m} \Omega / \mathrm{km}, 0.15 \mathrm{mH} / \mathrm{km}$

$V_{d c}$ nominal value: $1.2 \mathrm{kV}$

Both VSC filter: $C \_$filt $=8.9144 \mathrm{e}-04 \mathrm{~F}, L \_$filt $=1.1366 \mathrm{e}-06 \mathrm{~F}, R \_$filt $=0.0357 \Omega$

DC Terminal Capacitance $C_{c}, C_{3}, C_{6}: 4 \mathrm{mF}$

VSC reactance: 0.15 p.u. (based on $2 \mathrm{MW}$ )

VSC transformer inductance: 0.08 p.u.

$T_{3}: 0.017 \mathrm{~s}$

$T_{6}: 0.0072 \mathrm{~s}$

$T_{C}: 0.002 \mathrm{~s}$

$T_{G}: 0.002 \mathrm{~s}$

DC Voltage loop PI parameters: $K_{p V D C 3}: 40 \pi C_{3} ; K_{i V D C 3}: 400 \pi^{2} C_{3}$

$\mathrm{AC} \mathrm{d}$ and q axis Voltage Loops: $K_{p V A C}=180 \pi C_{-}$filt, $K_{i V D C}=8100 \pi^{2} C_{-}$filt

Line 36: $4 \mathrm{~km}$

LineG3: $1 \mathrm{~km}$

Line $3 \mathrm{C}: 2 \mathrm{~km}$

$R_{v C}: 0.72 \Omega$

$C_{v C}: 3.6 \mathrm{mF}$

Net Load (p.u.):

Feeder 1: $0.15+\mathrm{j} 0.05$

Feeder 2: $0.5+\mathrm{j} 0.01$

Feeder 3: $0.05+\mathrm{j} 0.01$

Feeder 4:-0.095+j0.001

Feeder 5: $-0.245+\mathrm{j} 0.001$

Feeder 6: $-0.15+\mathrm{j} 0.001$

Feeder 7: 0.005+j0.001

Feeder 8: 0.002+j0.004

Feeder 9: $-0.027+\mathrm{j} 0.003$ 

Institution of Engineering and Technology Copyright. The copy of record is available at IET Digital Library. 